\title{
Testing the equality of nonparametric regression curves based on Fourier coefficients
}

\author{
Zaher Mohdeb, Kenza Assia Mezhoud, and Djamel Boudaa \\ Department of Mathematics, Mentouri University, Constantine, Algeria
}

Received 7 July 2010; Accepted 30 September 2010

Copyright (C) 2010, Journal Afrika Statistika. All rights reserved

\begin{abstract}
In this work we propose a new methodology for the comparison of two regression functions $f_{1}$ and $f_{2}$ in the case of homoscedastic error structure and a fixed design. Our approach is based on the empirical Fourier coefficients of the regression functions $f_{1}$ and $f_{2}$ respectively. As our main results we obtain the asymptotic distribution of the test statistic under the null hypothesis $f_{1}=f_{2}$ and local and global alternatives. A simulation study is conducted to investigate the finite sample performance of our test.

Résumé. Dans ce travail, nous proposons une nouvelle méthode pour comparer deux fonctions de régression $f_{1}$ et $f_{2}$ dans le cas homoscédastique et d'un échantillonnage fixe. Notre approche est basée sur les coefficients de Fourier empiriques des fonctions de régression $f_{1}$ et $f_{2}$. Nous obtenons la distribution asymptotique de la statistique de test sous l'hypothèse nulle $f_{1}=f_{2}$ et les hypothèses alternatives ainsi que sous les hypothèses alternatives locales. Une étude par simulation est menée pour étudier la performance du test avec une petite taille d'échantillon.
\end{abstract}

Key words: Nonparametric regression; Test of equality, Empirical Fourier coefficient.

AMS 2010 Mathematics Subject Classification : 62G08, 62G10.

\section{Introduction}

The comparison of two regression curves is an important problem in applied regression analysis. In many applied fields, it has been of interest to choose between two regression functions of a response variable observed in two groups on an explanatory variable. Consider two regression models in fixed design given by

$$
Y_{l, j}=f_{l}\left(t_{j}\right)+\varepsilon_{l, j}, \quad l=1,2 \text { and } j=1, \ldots, n,
$$

where the design points $t_{j}$ are equispaced points and rescaled into the unit interval, $t_{j}=j / n ;$ for $l=1,2, f_{l}:[0,1] \longrightarrow$ $\mathbb{R}$, is unknown function and the errors $\left(\varepsilon_{l, j}\right)_{j=1, \ldots, n}$, are i.i.d. random variables with mean zero and variance $\sigma_{l}^{2}$. In addition, the random errors $\left(\varepsilon_{l, j}\right)_{j=1, \ldots, n}, l=1,2$ are assumed to be independent among themselves. In this paper, we are interested in the problem of testing the equality of the regression functions $f_{1}$ and $f_{2}$, that is,

$$
H_{0}: \quad f_{1}=f_{2} \quad \text { against } \quad H_{1}: \quad f_{1} \neq f_{2} .
$$

The problem of testing the equality of regression functions has been broadly studied in the recent literature. Some relevant papers are Härdle and Marron [9], Delgado [4], Young and Bowman [17], Bowman and Young [2], Hall [8], Kulasekera and Wang [10, 11], Munk and Dette [12] or Dette and Neumeyer [5]. Most of these works concentrate on equal design point and homoscedastic error. Cencov [3] studied the observation of an unknown distribution density from observations. Dette and Neumeyer [5] proposed several tests for hypothesis (2) which are based on kernel smoothing methods. Recently, Neumeyer and Dette [13] proposed a test for comparison of two regression curves that is based on

Zaher Mohdeb: z.mohdeb@gmail.com

Kenza Assia Mezhoud: m.kenzassia@yahoo.fr

Djamel Boudaa: dboudaa@yahoo.fr 
a difference of two marked empirical processes based on residuals. Vilar-Fernández and González-Mantega [15] studied the problem of checking the equality of $k$ regressions with dependent errors in a general context. Vilar-Fernández, Vilar-Fernández and González-Mantega [16] studied the problem of testing the equality of regression curves with dependent data using bootstrap algorithm to approximate the distribution of the test statistics.

Our aim in this paper is to construct hypothesis test (2) based on the Fourier coefficients of the regression functions $f_{1}$ and $f_{2}$. More precisely, let

$$
c_{l, k}=\int_{0}^{1} e^{-2 \pi i k t} f_{l}(t) d t, \quad l=1,2 \text { and } k \in \mathbb{Z}
$$

be the Fourier coefficients of $f_{l}, l=1,2$. The test (2) reduces to a test

$$
H_{0}: c_{1, k}=c_{2, k}, \forall k \in \mathbb{Z} \quad \text { against } \quad H_{1}: \exists k \in \mathbb{Z} \text { such that } c_{1, k} \neq c_{2, k} .
$$

Since for $l=1,2, f_{l}$ is assumed to be a real-valued function, we have $c_{l, k}=\bar{c}_{l,-k}$ for any $k \in \mathbb{N}$; consequently any test on the Fourier coefficients of $f_{l}$ reduces to a test on the $c_{l, k} k \geq 0$. In particular, the null hypothesis $H_{0}$ is equivalent to $c_{1, k}=c_{2, k},|k| \in \mathbb{N}$. It follows clearly that $H_{0}$ is true if and only if $\sum_{|k| \in \mathbb{N}}\left|c_{1, k}-c_{2, k}\right|^{2}=0$.

The test statistic we use, is based on the empirical estimators $\hat{c}_{l, k}$ of $c_{l, k}$ defined by

$$
\hat{c}_{l, k}=\frac{1}{n} \sum_{j=1}^{n} Y_{l, j} e^{-2 \pi i k j / n}, \quad l=1,2
$$

and we consider a sequence $p=p(n)$ of integers chosen such that $\lim _{n \rightarrow \infty} p(n)=\infty$. The test statistic is then based upon

$$
\hat{T}_{n, p}=\sum_{|k| \leq p}\left|\hat{c}_{1, k}-\hat{c}_{2, k}\right|^{2}
$$

and we reject $H_{0}$ if $\hat{T}_{n, p}>t$ for some $t>0$.

In our approach, the test statistic is used to test hypothesis on the Fourier coefficients of $f_{l}, l=1,2$. In the effective construction of the test, an estimation of the variance $\sigma_{l}^{2}, l=1,2$ is needed. This can be done in many ways. In the present paper, we consider the Rice [14] estimator. In order to study the power properties of the test, we conduct some Monte Carlo simulations.

The paper is organized as follows. In Section 2, we present the main results and Monte Carlo simulations. The proofs are deferred to Section 3.

\section{Assumptions and main results}

We are concerned with the test (3) for the model (1). Throughout this paper we assume for $l=1,2$.

$f_{l}$ satisfies the Lipschitz condition of order $\delta$, with $\frac{1}{2}<\delta \leq 1$, i.e. there exists a positive constant $M_{l}$ such that $\left|f_{l}(s)-f_{l}(t)\right| \leq M_{l}|s-t|^{\delta}$, for all $s, t \in[0,1]$.

$\varepsilon_{l, j}, j=1, \ldots, n$ are i.i.d. real random variables with zero mean and unknown variance $\sigma_{l}^{2}$.

The convergence in distribution will be denoted by $\stackrel{\mathcal{D}}{\longrightarrow}$ and the convergence in probability by $\stackrel{\mathcal{P}}{\longrightarrow}$. We introduce an increasing sequence $p(n)$ such that $\lim _{n \rightarrow+\infty} p(n)=+\infty$. In the results and proofs, we use the notation $p$ for the sequence $p(n)$.

\subsection{Asymptotic behaviour of the empirical Fourier coefficients}

We introduce in addition the following assumptions,

(A1) $\varepsilon_{l, 1} \sim \mathcal{N}\left(0, \sigma_{l}^{2}\right), l=1,2$.

(A2) $\lim _{n \rightarrow+\infty}\left\{n^{-2 \delta+1} p(n)\right\}=0$. 
Theorem 1. If $(A 1)$ and $(A 2)$ hold, then we have

$$
\frac{n \sum_{|k| \leq p}\left|\left(\hat{c}_{1, k}-\hat{c}_{2, k}\right)-\left(c_{1, k}-c_{2, k}\right)\right|^{2}-(2 p+1)\left(\sigma_{1}^{2}+\sigma_{2}^{2}\right)}{\left(\sigma_{1}^{2}+\sigma_{2}^{2}\right) \sqrt{2(2 p+1)}} \underset{n \rightarrow+\infty}{\stackrel{\mathcal{D}}{\longrightarrow}} \mathcal{N}(0,1) .
$$

Since the critical region is defined by $\hat{T}_{n, p}=\sum_{|k| \leq p}\left|\hat{c}_{1, k}-\hat{c}_{2, k}\right|^{2}>t$, Theorem 1 gives asymptotic level and power of the test when $\sigma_{1}^{2}$ and $\sigma_{2}^{2}$ are known. The question that arises in the nonparametric case is, what alternatives approaching $H_{0}$ can be distinguished from the null hypothesis $H_{0}$. Following Eubank and Spiegelman [6], we consider the local alternatives $c_{1, k}-c_{2, k}=h(n) e_{k},|k| \leq p$, where $\lim _{n \rightarrow \infty} h(n)=0$ and $e_{k}$ is the $k$-th Fourier coefficient of a function $m$ satisfying the Lipschitz condition of order $\delta>1 / 2$, we obtain:

Proposition 1. Let the assumptions of Theorem 1 hold, then for local alternatives $c_{1, k}-c_{2, k}=h(n) e_{k},|k| \leq p$, where $h(n)=p^{1 / 4} n^{-1 / 2}$, we have

$$
\frac{n \sum_{|k| \leq p}\left|\hat{c}_{1, k}-\hat{c}_{2, k}\right|^{2}-(2 p+1)\left(\sigma_{1}^{2}+\sigma_{2}^{2}\right)}{\left(\sigma_{1}^{2}+\sigma_{2}^{2}\right) \sqrt{2(2 p+1)}} \underset{n \rightarrow+\infty}{\stackrel{\mathcal{D}}{\longrightarrow}} \mathcal{N}\left(\frac{1}{2\left(\sigma_{1}^{2}+\sigma_{2}^{2}\right)} \sum_{|k| \in \mathbb{N}}\left|e_{k}\right|^{2}, 1\right) .
$$

Proposition 1 means that the test can detect local alternatives converging to the null hypothesis at the rate of $p^{1 / 4} n^{-1 / 2}$ or slower.

\subsection{Construction of the tests}

The result of Theorem 1 can be used to construct the test of the hypothesis $H_{0}$ in (3); however in practice the variances $\sigma_{1}^{2}$ and $\sigma_{2}^{2}$ are unknown and thus we have to estimate them. We can use the estimator given by Gasser, Sroka and Jennen-Steinmetz [7]; in this work, we use the Rice [14] estimator

$$
\hat{\sigma}_{l}^{2}=\frac{1}{2(n-1)} \sum_{j=2}^{n}\left(Y_{l, j}-Y_{l, j-1}\right)^{2}, \quad l=1,2 .
$$

Under our assumptions, we can easily prove that $\hat{\sigma}_{l}^{2}$ converges to $\sigma_{l}^{2}$ at the rate $n^{-1 / 2}$ and Theorem 1 remains valid with $\hat{\sigma}_{l}^{2}$ instead of $\sigma_{l}^{2}, l=1,2$.

Corollary 1. If (A1) and (A2) hold, then under the null hypothesis $H_{0}: f_{1}=f_{2}$, we have

$$
\frac{n \sum_{|k| \leq p}\left|\hat{c}_{1, k}-\hat{c}_{2, k}\right|^{2}-(2 p+1)\left(\hat{\sigma}_{1}^{2}+\hat{\sigma}_{2}^{2}\right)}{\left(\hat{\sigma}_{1}^{2}+\hat{\sigma}_{2}^{2}\right) \sqrt{2(2 p+1)}} \underset{n \rightarrow+\infty}{\stackrel{\mathcal{D}}{\longrightarrow}} \mathcal{N}(0,1) .
$$

These results give the level of significance when the regression functions satisfy the Lipschitz condition under the null hypothesis, and the power for alternatives which satisfy also the Lipschitz condition. In the next section we use Monte Carlo methods to study power of our test.

\subsection{Simulation}

In order to investigate the power of our test, we conducted a small scale simulation using the models $Y_{l, j}=f_{l}\left(t_{j}\right)+\varepsilon_{l, j}$, $j=1, \ldots, n$, with $t_{j}=j / n$ and the $\left(\varepsilon_{l, j}\right)_{j=1, \ldots, n}$ are i.i.d. random errors distributed as $\mathcal{N}\left(0, \sigma_{l}^{2}\right)$, where $\sigma_{l}=0.25,0.50$, $l=1,2$.

In our simulations, we study the test of the hypothesis

$$
H_{0}: f_{1}=f_{2} \quad \text { against } \quad H_{1}: f_{1} \neq f_{2} .
$$

Our test statistic is

$$
\hat{T}_{p}=\frac{n \sum_{|k| \leq p}\left|\hat{c}_{1, k}-\hat{c}_{2, k}\right|^{2}-(2 p+1)\left(\hat{\sigma}_{1}^{2}+\hat{\sigma}_{2}^{2}\right)}{\left(\hat{\sigma}_{1}^{2}+\hat{\sigma}_{2}^{2}\right) \sqrt{2(2 p+1)}},
$$

where $\hat{c}_{l, k}$ are the empirical coefficients of $f_{l}$ and $\hat{\sigma}_{l}^{2}$ is the Rice [14] estimator given in (5) for $l=1,2$. 
The Monte Carlo study, for sample size $n=100$, turns on the comparison of the empirical power test statistic $\hat{T}_{p}$ and the test statistic $\hat{M}^{2}$ proposed by Munk and Dette [12].

The hypothesis $H_{0}$ is rejected if $\hat{T}_{p}>t_{1-\alpha}$ and $\frac{\sqrt{n} \hat{M}^{2}}{\hat{\sigma}_{1}^{2}+\hat{\sigma}_{2}^{2}}>t_{1-\alpha}$ respectively in our situation, where $t_{1-\alpha}$ is the $(1-\alpha)$ quantile of the standard normal distribution.

In Table 1 and Table 2, we test for the validity of the null hypothesis $H_{0}$ with: $f_{1}(t)=t$ and two different forms for $f_{2}: f_{2}^{(1)}(t)=\beta t e^{-2 t}$ and $f_{2}^{(2)}(t)=\beta t^{2}$ respectively, for several choices of $\beta$ on the interval [0,2]. The test statistics are computed under all combinations of the foregoing factors and is used to decide whether or not to reject the null hypothesis at the level $\alpha=0.05$. Empirical critical values are used in our power study. The empirical powers of $\hat{T}_{p}$ and $\hat{M}^{2}$ are denoted by $P_{T}$ and $P_{M D}$ respectively.

For each combination of the factors, we replicat the experiment 1000 times and record the proportion of rejections. It appears that the two empirical powers denoted respectively by $P_{T}$ and $P_{M D}$ are quite similar. We note that the power decreases when the variance of the noise increases; this is not surprising, since the signal is drowned in the noise. In our simulations $p=5$, because we note that the value $p=5\left(<n^{1 / 3}\right)$ works better than the other values of $p$; the optimal selection of a value for $p$ in the test remains an unsolved problem.

\begin{tabular}{|c|c|c|c|c|c|c|c|c|}
\hline \multirow[b]{2}{*}{$\beta$} & \multicolumn{2}{|c|}{$\begin{array}{l}\sigma_{1}=0.25 \\
\sigma_{2}=0.25\end{array}$} & \multicolumn{2}{|c|}{$\begin{array}{l}\sigma_{1}=0.25 \\
\sigma_{2}=0.50\end{array}$} & \multicolumn{2}{|c|}{$\begin{array}{l}\sigma_{1}=0.50 \\
\sigma_{2}=0.25\end{array}$} & \multicolumn{2}{|c|}{$\begin{array}{l}\sigma_{1}=0.50 \\
\sigma_{2}=0.50\end{array}$} \\
\hline & $P_{T}$ & $P_{M D}$ & $P_{T}$ & $P_{M D}$ & $P_{T}$ & $P_{M D}$ & $P_{T}$ & $P_{M D}$ \\
\hline 0.0 & 0.046 & 0.055 & 0.043 & 0.052 & 0.046 & 0.060 & 0.048 & 0.053 \\
\hline 0.1 & 0.058 & 0.052 & 0.051 & 0.064 & 0.055 & 0.055 & 0.044 & 0.057 \\
\hline 0.5 & 0.221 & 0.162 & 0.124 & 0.095 & 0.113 & 0.106 & 0.086 & 0.074 \\
\hline 1.0 & 0.851 & 0.533 & 0.373 & 0.217 & 0.372 & 0.210 & 0.237 & 0.134 \\
\hline 1.5 & 0.998 & 0.934 & 0.772 & 0.473 & 0.780 & 0.460 & 0.541 & 0.336 \\
\hline 2.0 & 1.000 & 1.000 & 0.965 & 0.803 & 0.963 & 0.787 & 0.819 & 0.545 \\
\hline
\end{tabular}

Table 1. Proportion of rejections in 1000 samples of size $n=100$, with $f_{1}(t)=t$ and $f_{2}(t)=t+\beta t e^{-2 t}$.

\begin{tabular}{|c|c|c|c|c|c|c|c|c|}
\hline \multirow[b]{2}{*}{$\beta$} & \multicolumn{2}{|c|}{$\begin{array}{l}\sigma_{1}=0.25 \\
\sigma_{2}=0.25\end{array}$} & \multicolumn{2}{|c|}{$\begin{array}{l}\sigma_{1}=0.25 \\
\sigma_{2}=0.50\end{array}$} & \multicolumn{2}{|c|}{$\begin{array}{l}\sigma_{1}=0.50 \\
\sigma_{2}=0.25\end{array}$} & \multicolumn{2}{|c|}{$\begin{array}{l}\sigma_{1}=0.50 \\
\sigma_{2}=0.50\end{array}$} \\
\hline & $P_{T}$ & $P_{M D}$ & $P_{T}$ & $P_{M D}$ & $P_{T}$ & $P_{M D}$ & $P_{T}$ & $P_{M D}$ \\
\hline 0.0 & 0.052 & 0.059 & 0.042 & 0.055 & 0.052 & 0.049 & 0.048 & 0.049 \\
\hline 0.1 & 0.103 & 0.094 & 0.076 & 0.077 & 0.069 & 0.082 & 0.055 & 0.058 \\
\hline 0.5 & 0.996 & 0.938 & 0.725 & 0.473 & 0.730 & 0.470 & 0.495 & 0.306 \\
\hline 1.0 & 1.000 & 1.000 & 1.000 & 0.992 & 1.000 & 0.995 & 0.997 & 0.919 \\
\hline 1.5 & 1.000 & 1.000 & 1.000 & 1.000 & 1.000 & 1.000 & 1.000 & 1.000 \\
\hline 2.0 & 1.000 & 1.000 & 1.000 & 1.000 & 1.000 & 1.000 & 1.000 & 1.000 \\
\hline
\end{tabular}

Table 2. Proportion of rejections in 1000 samples of size $n=100$, with $f_{1}(t)=t$ and $f_{2}(t)=t+\beta t^{2}$.

\section{Proofs}

Let us denote, $\forall k \in \mathbb{Z}$,

$$
\mu_{k}=c_{1, k}-c_{2, k}, \quad \tilde{\mu}_{k}=\tilde{c}_{1, k}-\tilde{c}_{2, k}, \quad \text { and } \quad \hat{\mu}_{k}=\hat{c}_{1, k}-\hat{c}_{2, k},
$$


where $\tilde{c}_{l, k}(l=1,2)$ is the $k$-th discrete Fourier coefficient of the regression function $f_{l}$ defined by

$$
\tilde{c}_{l, k}=\frac{1}{n} \sum_{j=1}^{n} f_{l}(j / n) e^{-2 \pi i j k / n}, \quad l=1,2 .
$$

We have

$$
\left(\hat{c}_{1, k}-\hat{c}_{2, k}\right)-\left(\tilde{c}_{1, k}-\tilde{c}_{2, k}\right)=\alpha_{k}-i \beta_{k},
$$

where

$$
\alpha_{k}=\frac{1}{n} \sum_{j=1}^{n} \xi_{j} \cos (2 \pi j k / n) \quad \text { and } \quad \beta_{k}=\frac{1}{n} \sum_{j=1}^{n} \xi_{j} \sin (2 \pi j k / n)
$$

with

$$
\xi_{j}=\varepsilon_{1, j}-\varepsilon_{2, j}, \quad j=1, \ldots, n .
$$

Note that $E\left(\hat{c}_{l, k}\right)=\tilde{c}_{l, k}, l=1,2$.

Lemma 1. For $\alpha_{k}$ and $\beta_{k}$ defined in (9), we have

$$
\begin{gathered}
\operatorname{Var}\left(\alpha_{k}\right)=E\left(\alpha_{k}^{2}\right)= \begin{cases}\left(\sigma_{1}^{2}+\sigma_{2}^{2}\right) /(2 n) & \text { if } 0<|k|<n / 2 \\
\left(\sigma_{1}^{2}+\sigma_{2}^{2}\right) / n & \text { if } k=0 \text { or }|k|=n / 2\end{cases} \\
\operatorname{Var}\left(\beta_{k}\right)=E\left(\beta_{k}^{2}\right)= \begin{cases}\left(\sigma_{1}^{2}+\sigma_{2}^{2}\right) /(2 n) & \text { if } 0<|k|<n / 2 \\
0 & \text { if } k=0 \text { or }|k|=n / 2\end{cases} \\
\operatorname{Cov}\left(\alpha_{k}, \beta_{k^{\prime}}\right)=0 \quad \forall k \in \mathbb{Z}, k^{\prime} \in \mathbb{Z} \\
\operatorname{Cov}\left(\alpha_{k}, \alpha_{k^{\prime}}\right)=\operatorname{Cov}\left(\beta_{k}, \beta_{k^{\prime}}\right)=0 \text { if }|k| \neq\left|k^{\prime}\right| .
\end{gathered}
$$

\section{Proof of Lemma 1.}

The result follows from orthogonality of the cosine and sine functions.

Now, we start the proof of Theorem 1; we begin with the following Lemma.

Lemma 2. If $(A 1)$ holds, then

$$
\frac{n \sum_{|k| \leq p}\left|\left(\hat{c}_{1, k}-\hat{c}_{2, k}\right)-\left(\tilde{c}_{1, k}-\tilde{c}_{2, k}\right)\right|^{2}-(2 p+1)\left(\sigma_{1}^{2}+\sigma_{2}^{2}\right)}{\left(\sigma_{1}^{2}+\sigma_{2}^{2}\right) \sqrt{2(2 p+1)}} \underset{n \rightarrow+\infty}{\stackrel{\mathcal{D}}{\rightarrow}} \mathcal{N}(0,1) .
$$

\section{Proof of Lemma 2.}

Let us consider a sequence of integers $p(n)$ such that $\lim _{n \rightarrow+\infty} p(n)=+\infty$.

Set

$$
T_{n, p}=\frac{n \hat{\Lambda}_{n, p}-(2 p+1)\left(\sigma_{1}^{2}+\sigma_{2}^{2}\right)}{\left(\sigma_{1}^{2}+\sigma_{2}^{2}\right) \sqrt{2(2 p+1)}}
$$

where

$$
\hat{\Lambda}_{n, p}=\sum_{|k| \leq p}\left|\left(\hat{c}_{1, k}-\tilde{c}_{1, k}\right)-\left(\hat{c}_{2, k}-\tilde{c}_{2, k}\right)\right|^{2} .
$$

Let $\alpha_{k}$ and $\beta_{k}$ be given by (9). In view of Lemma 1 and assumption (A1), we have

$$
\sqrt{n}\left(\alpha_{0}, \sqrt{2} \alpha_{1}, \sqrt{2} \beta_{1}, \ldots, \sqrt{2} \alpha_{p}, \sqrt{2} \beta_{p}\right)^{\prime} \sim \mathcal{N}\left(0,\left(\sigma_{1}^{2}+\sigma_{2}^{2}\right) I_{2 p+1}\right),
$$

where $I_{2 p+1}$ is the $(2 p+1) \times(2 p+1)$ identity matrix.

Then

$$
\frac{n}{\sigma_{1}^{2}+\sigma_{2}^{2}} \hat{\Lambda}_{n, p}=\frac{n}{\sigma_{1}^{2}+\sigma_{2}^{2}}\left[\alpha_{0}^{2}+2 \sum_{k=1}^{p}\left(\alpha_{k}^{2}+\beta_{k}^{2}\right)\right] \sim \chi^{2}(2 p+1) .
$$


We know, from the central limit theorem, that if $\left(Z_{i}\right)_{i \geq 1}$ is a sequence of i.i.d. $\chi^{2}(1)$ distributed random variables, we have

$$
\frac{S_{n, p}-(2 p+1)}{\sqrt{2(2 p+1)}} \underset{n \rightarrow+\infty}{\stackrel{\mathcal{D}}{\longrightarrow}} \mathcal{N}(0,1)
$$

where $S_{n, p}=\sum_{k=1}^{2 p+1} Z_{k}$.

Since $\frac{n}{\sigma_{1}^{2}+\sigma_{2}^{2}} \hat{\Lambda}_{n, p}$ and $S_{n, p}$ have the same law for all $n$, then

$$
\frac{\frac{n}{\sigma_{1}^{2}+\sigma_{2}^{2}} \hat{\Lambda}_{n, p}-(2 p+1)}{\sqrt{2(2 p+1)}} \underset{n \rightarrow+\infty}{\stackrel{\mathcal{D}}{\longrightarrow}} \mathcal{N}(0,1) .
$$

Lemma 2 is then proved.

\section{Proof of Theorem 1.}

According to (6), we have

$$
\left|\left(\hat{c}_{1, k}-\hat{c}_{2, k}\right)-\left(c_{1, k}-c_{2, k}\right)\right|^{2}=\left|\left(\hat{\mu}_{k}-\tilde{\mu}_{k}\right)+\left(\tilde{\mu}_{k}-\mu_{k}\right)\right|^{2}
$$

and then

$$
\frac{n \sum_{|k| \leq p}\left|\left(\hat{c}_{1, k}-\hat{c}_{2, k}\right)-\left(c_{1, k}-c_{2, k}\right)\right|^{2}-(2 p+1)\left(\sigma_{1}^{2}+\sigma_{2}^{2}\right)}{\left(\sigma_{1}^{2}+\sigma_{2}^{2}\right) \sqrt{2(2 p+1)}}=Z_{n}+U_{n}+V_{n}
$$

where

$$
\begin{gathered}
Z_{n}=\frac{n \sum_{|k| \leq p}\left|\hat{\mu}_{k}-\tilde{\mu}_{k}\right|^{2}-(2 p+1)\left(\sigma_{1}^{2}+\sigma_{2}^{2}\right)}{\left(\sigma_{1}^{2}+\sigma_{2}^{2}\right) \sqrt{2(2 p+1)}} \\
U_{n}=\frac{n}{\left(\sigma_{1}^{2}+\sigma_{2}^{2}\right) \sqrt{2(2 p+1)}} \sum_{|k| \leq p}\left|\tilde{\mu}_{k}-\mu_{k}\right|^{2} \\
V_{n}=\frac{n}{\left(\sigma_{1}^{2}+\sigma_{2}^{2}\right) \sqrt{2(2 p+1)}} \sum_{|k| \leq p}\left[\left(\hat{\mu}_{k}-\tilde{\mu}_{k}\right)\left(\overline{\tilde{\mu}_{k}-\mu_{k}}\right)+\left(\overline{\hat{\mu}_{k}-\tilde{\mu}_{k}}\right)\left(\tilde{\mu}_{k}-\mu_{k}\right)\right] .
\end{gathered}
$$

According to Lemma 2 , to prove the result, it is sufficient to show that $\lim _{n \rightarrow \infty} U_{n}=0$ and $V_{n} \stackrel{\mathcal{P}}{\longrightarrow} 0$ as $n \rightarrow \infty$.

We have

$$
\begin{aligned}
\left|\tilde{\mu}_{k}-\mu_{k}\right| & =\left|\frac{1}{n} \sum_{j=1}^{n}\left(f_{1}-f_{2}\right)(j / n) e^{-2 \pi i j k / n}-\int_{0}^{1}\left(f_{1}-f_{2}\right)(t) e^{-2 \pi i k t} d t\right| \\
& \leq \sum_{l=1}^{2} \sum_{j=1}^{n} \int_{(j-1) / n}^{j / n}\left|f_{l}(j / n) e^{-2 \pi i j k / n}-f_{l}(t) e^{-2 \pi i k t}\right| d t .
\end{aligned}
$$

Since $f_{l}(l=1,2)$ satisfies the Lipschitz condition of order $\delta$ and the exponential function is continuously differentiable, it is easy to prove that there exists constants $M_{l}>0$ such that

$$
\left|f_{l}(j / n) e^{-2 \pi i j k / n}-f_{l}(t) e^{-2 \pi i k t}\right| \leq M_{l}\left(\frac{j}{n}-t\right)^{\delta}, \quad l=1,2 .
$$

It follows that

$$
\int_{(j-1) / n}^{j / n}\left|f_{l}(j / n) e^{2 \pi i j k / n}-f_{l}(t) e^{2 \pi i k t}\right| d t \leq \frac{M_{l}}{(\delta+1) n^{\delta+1}}, \quad l=1,2 .
$$

Therefore

$$
\left|\tilde{\mu}_{k}-\mu_{k}\right| \leq \frac{M}{(\delta+1) n^{\delta}}, \quad \text { where } \quad M=M_{1}+M_{2}
$$

It follows from (13) that

$$
U_{n} \leq \frac{M^{2}}{(\delta+1)^{2}\left(\sigma_{1}^{2}+\sigma_{2}^{2}\right) n^{2 \delta-1}} \sqrt{\frac{2 p+1}{2}} .
$$

Then, using assumption (A2), we have $\lim _{n \rightarrow \infty} U_{n}=0$. 
Now,

$$
E\left|V_{n}\right| \leq \frac{2 n}{\left(\sigma_{1}^{2}+\sigma_{2}^{2}\right) \sqrt{2(2 p+1)}} \sum_{|k| \leq p}\left|\tilde{\mu}_{k}-\mu_{k}\right| E\left|\hat{\mu}_{k}-\tilde{\mu}_{k}\right| .
$$

In view of (8) and Lemma 1, we have

$$
E\left|\hat{\mu}_{k}-\tilde{\mu}_{k}\right|^{2}=E\left(\alpha_{k}^{2}\right)+E\left(\beta_{k}^{2}\right)=\frac{\sigma_{1}^{2}+\sigma_{2}^{2}}{n}
$$

and thus

$$
E\left|\hat{\mu}_{k}-\tilde{\mu}_{k}\right| \leq \sqrt{\frac{\sigma_{1}^{2}+\sigma_{2}^{2}}{n}} .
$$

According to (13) and (14), we deduce that

$$
E\left|V_{n}\right| \leq \frac{M \sqrt{2(2 p+1)}}{(\delta+1) n^{\delta-\frac{1}{2}} \sqrt{\sigma_{1}^{2}+\sigma_{2}^{2}}}
$$

Then, using assumption (A2), we deduce that $V_{n} \stackrel{\mathcal{P}}{\longrightarrow} 0$ as $n \rightarrow \infty$.

Remark 1. As a consequence of (13) and (14), we note that

$$
E\left|\hat{\mu}_{k}-\mu_{k}\right|^{2} \leq \frac{C}{n}
$$

where the constant $C$ is given by $C=\sigma_{1}^{2}+\sigma_{2}^{2}+\frac{M^{2}}{(\delta+1)^{2}}+\frac{2 M \sqrt{\sigma_{1}^{2}+\sigma_{2}^{2}}}{\delta+1}$.

\section{Proof of Proposition 1.}

Since $c_{1, k}-c_{2, k}=h(n) e_{k}$ and according to (6), we have

$$
\hat{c}_{1, k}-\hat{c}_{2, k}=\hat{\mu}_{k}-\mu_{k}+h(n) e_{k}
$$

and

$$
\frac{n \sum_{|k| \leq p}\left|\hat{c}_{1, k}-\hat{c}_{2, k}\right|^{2}-(2 p+1)\left(\sigma_{1}^{2}+\sigma_{2}^{2}\right)}{\left(\sigma_{1}^{2}+\sigma_{2}^{2}\right) \sqrt{2(2 p+1)}}=F_{n}+G_{n}+H_{n},
$$

where

$$
\begin{gathered}
F_{n}=\frac{n \sum_{|k| \leq p}\left|\hat{\mu}_{k}-\mu_{k}\right|^{2}-(2 p+1)\left(\sigma_{1}^{2}+\sigma_{2}^{2}\right)}{\left(\sigma_{1}^{2}+\sigma_{2}^{2}\right) \sqrt{2(2 p+1)}} \\
G_{n}=\frac{n(h(n))^{2}}{\left(\sigma_{1}^{2}+\sigma_{2}^{2}\right) \sqrt{2(2 p+1)}} \sum_{|k| \leq p}\left|e_{k}\right|^{2}
\end{gathered}
$$

and

$$
H_{n}=\frac{n h(n)}{\left(\sigma_{1}^{2}+\sigma_{2}^{2}\right) \sqrt{2(2 p+1)}} \sum_{|k| \leq p}\left\{\left(\hat{\mu}_{k}-\mu_{k}\right) \overline{e_{k}}+\overline{\left(\hat{\mu}_{k}-\mu_{k}\right)} e_{k}\right\}
$$

According to Theorem 1 , we have $F_{n} \underset{n \rightarrow+\infty}{\stackrel{\mathcal{D}}{\rightarrow}} \mathcal{N}(0,1)$ and since $h(n)=\left(p^{1 / 4}\right) / \sqrt{n}$, we have $\lim _{n \rightarrow \infty} G_{n}=$ $\frac{1}{2\left(\sigma_{1}^{2}+\sigma_{2}^{2}\right)} \sum_{|k| \in \mathbb{N}}\left|e_{k}\right|^{2}$. Then, in order to prove our result, it suffices to show that $\lim _{n \rightarrow+\infty} E\left|H_{n}\right|=0$.

We have

$$
E\left|H_{n}\right| \leq \frac{2 n h(n)}{\left(\sigma_{1}^{2}+\sigma_{2}^{2}\right) \sqrt{2(2 p+1)}} \sum_{|k| \leq p}\left|e_{k}\right| E\left|\hat{\mu}_{k}-\mu_{k}\right|
$$

Since $m$ satisfies the Lipschitz condition of order $\delta>1 / 2\left(m\right.$ is not assumed to be periodic), we claim that $\sum_{k=0}^{\infty}\left|e_{k}\right|^{r}<$ $\infty, \forall r>1$. To prove this, we extend $m$ as a periodic function on $\mathbb{R}$ which satisfies the Lipschitz condition of order 
$\delta>1 / 2$ on each interval $[n, n+1]$ (but not on $\mathbb{R}$ ). The Lipschitz condition implies that $m$ is bounded. We have for $0<h<1$,

$$
\begin{aligned}
\int_{0}^{1}[m(t+h)-m(t-h)]^{2} d t= & \int_{h}^{1-h}[m(t+h)-m(t-h)]^{2} d t \\
& +\int_{0}^{h}[m(t+h)-m(t-h)]^{2} d t \\
& +\int_{1-h}^{1}[m(t+h)-m(t-h)]^{2} d t \\
\leq & C_{1} h^{2 \delta}+2 h \sup _{t \in[0,1]}|m(t)|,
\end{aligned}
$$

where $C_{1}$ is constant. Since $\delta>1 / 2$, we obtain

$$
\int_{0}^{1}[m(t+h)-m(t-h)]^{2} d t \leq C_{2} h,
$$

where $C_{2}$ is another constant.

Now following the proof in Bary [1, page 216], we obtain

$$
\left(\sum_{k=n}^{\infty}\left|e_{k}\right|^{r}\right)^{1 / r} \leq \frac{C}{n^{\delta+\frac{1}{2}-\frac{1}{r}}}
$$

Thus for $r>1$ and $\delta>1 / 2$, we have $\sum_{k=0}^{\infty}\left|e_{k}\right|^{r}<\infty$ and the claim is proved.

Let us return now to (16). Set $1<r<\frac{4}{3}$ and $s$ such that $\frac{1}{r}+\frac{1}{s}=1$.

Clearly $\frac{1}{s}<\frac{1}{4}$. Applying Hölder's inequality in (16), we obtain

$$
E\left|H_{n}\right| \leq \frac{2 n h(n)}{\left(\sigma_{1}^{2}+\sigma_{2}^{2}\right) \sqrt{2(2 p+1)}}\left(\sum_{|k| \leq p}\left|e_{k}\right|^{r}\right)^{1 / r}\left(\sum_{|k| \leq p}\left(E\left|\hat{\mu}_{k}-\mu_{k}\right|\right)^{s}\right)^{1 / s} .
$$

Since $h(n)=p^{1 / 4} / \sqrt{n}$ and according to (15), we have

$$
\begin{aligned}
E\left|H_{n}\right| & =O\left(\frac{n h(n)}{\sqrt{p}} \frac{p^{1 / s}}{\sqrt{n}}\right) \\
& =O\left(p^{\frac{1}{s}-\frac{1}{4}}\right) .
\end{aligned}
$$

Since $\frac{1}{s}<\frac{1}{4}$, we obtain $\lim _{n \rightarrow \infty} E\left|H_{n}\right|=0$. Proposition 1 is proved.

We start now the proof of the corollary.

\section{Proof of Corollary 1.}

We have,

$$
\begin{aligned}
& \frac{n \sum_{|k| \leq p}\left|\hat{c}_{1, k}-\hat{c}_{2, k}\right|^{2}-(2 p+1)\left(\hat{\sigma}_{1}^{2}+\hat{\sigma}_{2}^{2}\right)}{\left(\hat{\sigma}_{1}^{2}+\hat{\sigma}_{2}^{2}\right) \sqrt{2(2 p+1)}}= \\
& \frac{\sigma_{1}^{2}+\sigma_{2}^{2}}{\hat{\sigma}_{1}^{2}+\hat{\sigma}_{2}^{2}}\left\{\frac{n \sum_{|k| \leq p}\left|\hat{c}_{1, k}-\hat{c}_{2, k}\right|^{2}-(2 p+1)\left(\sigma_{1}^{2}+\sigma_{2}^{2}\right)}{\left(\sigma_{1}^{2}+\sigma_{2}^{2}\right) \sqrt{2(2 p+1)}}+\frac{\sqrt{2 p+1}\left[\left(\sigma_{1}^{2}+\sigma_{2}^{2}\right)-\left(\hat{\sigma}_{1}^{2}+\hat{\sigma}_{2}^{2}\right)\right]}{\sqrt{2}\left(\sigma_{1}^{2}+\sigma_{2}^{2}\right)}\right\} .
\end{aligned}
$$

Corollary 1 follows then from Theorem 1 . 


\section{References}

[1] Bary, N.K., 1964. A Treatise on Trigonometric Series. Pergamon Press.

[2] Bowman, A. and Young, S., 1996. Graphical comparison of nonparametric curves. Appl. Statist., 45, 83-98.

[3] Cencov, N.N. 1962. Evaluation of an unknown distribution density from observations. Soviet Math., 3, $1559-1562$.

[4] Delgado, M.A., 1993. Testing the equality of nonparametric regression curves. Statist. Probab. Lett., 17, $199-204$.

[5] Dette, H. and Neumeyer, N., 2001. Nonparametric analysis of covariance. Ann. Statist., 29, 1361-1400.

[6] Eubank, R.L. and Spiegelman, C.H., 1990. Testing the goodness-of-fit of a linear model via nonparametric regression techniques. J. Amer. Stat. Assoc., 85, 410, 387-392.

[7] Gasser, T., Sroka, L. and Jennen-Steinmetz, C., 1986. Residual variance and residual pattern in nonlinear regression. Biometrika, 73, 625-633.

[8] Hall, P., Huber, C. and Speckman, P.L., 1997. Covariate-matched one-sided tests for the difference between functional means. J. Amer. Statist. Assoc., 92, 1074-1083.

[9] Härdle, W. and Marron, J.S., 1990. Semiparametric comparison of regression curves. Ann. Statist., 18, 63-89.

[10] Kulasekera, K.B. and Wang, J., 1997. Smoothing parameter selection for power optimality in testing of regression curves. J. Amer. Statist. Assoc., 92(438), 500-511.

[11] Kulasekera, K.B. and Wang, J., 1998. Bandwidth selection for power optimality in a test of equality of regression curves. Stat. Probab. Lett., 37, 287-293.

[12] Munk, A. and Dette, H., 1998. Nonparametric comparison of several regression functions: Exact and asymptotic theory. Ann. Statist., 26, 2339-2368.

[13] Neumeyer, N. and Dette, H., 2003. Nonparametric comparison of regression curves: an empirical process approach. Ann. Statist., 31, 3, 880-920.

[14] Rice, J.A., 1984. Bandwidth choice for nonparametric regression. Ann. Statist., 12, 1215-1230.

[15] Vilar-Fernández, J.M. and González-Manteiga, W., 2003. Nonparametric comparison of curves with dependent errors. Statistics, 38, 2, 81-99.

[16] Vilar-Fernández, J.M., Vilar-Fernández, J.A. and González-Manteiga, W., 2007. Bootstrap tests for nonparametric comparison of regression curves with dependent errors. TEST, 16, 123-144.

[17] Young, S.G. and Bowman, A.W., 1995. Nonparametric analysis of covariance. Biometrics. 51, 920-931. 